\title{
Enhanced Intersystem Crossing and Transient Electron Spin Polarization in a Photoexcited Pentacene-Trityl Radical
}

\author{
Claudia E. Avalos, Sabine Richert, Etienne Socie, Ganesan Karthikeyan, Gilles Casano, \\ Gabriele Stevanato, Dominik J. Kubicki, Jacques E. Moser, Christiane R. Timmel, Moreno Lelli, \\ Aaron J. Rossini, Olivier Ouari,* and Lyndon Emsley*
}

Cite This: J. Phys. Chem. A 2020, 124, 6068-6075

Read Online

ACCESS | Lلll Metrics \& More | 回 Article Recommendations ｜ st Supporting Information

ABSTRACT: Identifying and characterizing systems that generate well-defined states with large electron spin polarization is of high interest for applications in molecular spintronics, high-energy physics, and magnetic resonance spectroscopy. The generation of electron spin polarization on free-radical substituents tethered to pentacene derivatives has recently gained a great deal of interest for its applications in molecular electronics. After photoexcitation of the chromophore, pentacene-radical derivatives can rapidly form spinpolarized triplet excited states through enhanced intersystem crossing. Under the right conditions, the triplet spin polarization, arising from $m_{\mathrm{S}}$-selective intersystem crossing rates, can be transferred to the tethered stable radical. The efficiency of this spin polarization transfer depends on many factors: local magnetic and electric fields, excited-

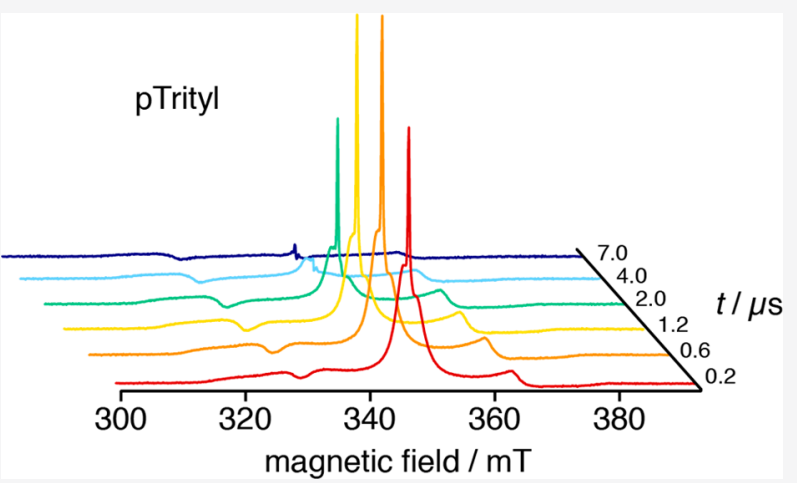
state energetics, molecular geometry, and spin-spin coupling. Here, we present transient electron paramagnetic resonance (EPR) measurements on three pentacene derivatives tethered to Finland trityl, BDPA, or TEMPO radicals to explore the influence of the nature of the radical on the spin polarization transfer. We observe efficient polarization transfer between the pentacene excited triplet and the trityl radical but do not observe the same for the BDPA and TEMPO derivatives. The polarization transfer behavior in the pentacene-trityl system is also investigated in different glassy matrices and is found to depend markedly on the solvent used. The EPR results are rationalized with the help of femtosecond and nanosecond transient absorption measurements, yielding complementary information on the excited-state dynamics of the three pentacene derivatives. Notably, we observe a 2 orders of magnitude difference in the time scale of triplet formation between the pentacene-trityl system and the pentacene systems tethered with the BDPA and TEMPO radicals.

\section{INTRODUCTION}

Stable radical-chromophore open-shell hybrids have emerged as a versatile class of molecules for light emission, ${ }^{1-3}$ chemical sensing, ${ }^{4}$ information storage, ${ }^{5}$ and studying the fundamental photophysics of processes such as spin catalysis. ${ }^{6,7}$ In particular, the ability to spin polarize radicals by optical pumping of the coupled chromophore has allowed one to considerably increase the sensitivity of select electron spin resonance experiments. ${ }^{8-10}$ In the last few decades there has been considerable progress in the understanding of optically induced spin polarization transfer and charge transfer mechanisms in photoactive molecules ${ }^{11,12}$ using model systems such as $\mathrm{C}_{60}-$ TEMPO, ${ }^{8,13,14}$ naphthaleneTEMPO, ${ }^{15}$ NDI-TEMPO, ${ }^{12,16}$ ZnTPP-BDPA, ${ }^{17}$ NDI$\mathrm{BDPA}^{18}$ and pentacene-TEMPO. ${ }^{6}$ In parallel, an increasing number of these studies have focused on closed- and open-shell pentacene derivatives with the goal of increasing their stability, solubility, and triplet yield. ${ }^{19-22}$ An increased triplet yield has, for example, implications for improving charge carrier mobilities in pentacene-doped solar cell devices ${ }^{23}$ and applications in molecular spintronics, artificial photosynthesis, and signal enhancement methods for magnetic resonance. ${ }^{24-28}$ The triplet yield can be enhanced by increasing the intersystem crossing rate, which may be accomplished by addition of free-radical substituents. ${ }^{19}$

Addition of a radical substituent can also lead to spin polarization transfer from the chromophore to the radical upon light irradiation, resulting in a significant increase or decrease in the observed electron paramagnetic resonance (EPR) signal of the radical substituent. $6,18,29,30$

The efficiency and the mechanism of spin polarization transfer are dictated by the type of chromophore used, the nature of the

Received: April 20, 2020

Revised: June 25, 2020

Published: June 25, 2020 
tethered radical, as well as the geometry, rigidity, and extent of conjugation of the linker. $6,29,31$

In order to explore the effect of the radical on the efficiency and mechanism of the polarization transfer between the pentacene and the tethered radical, we synthesized and characterized three pentacene derivatives, shown in Figure 1,
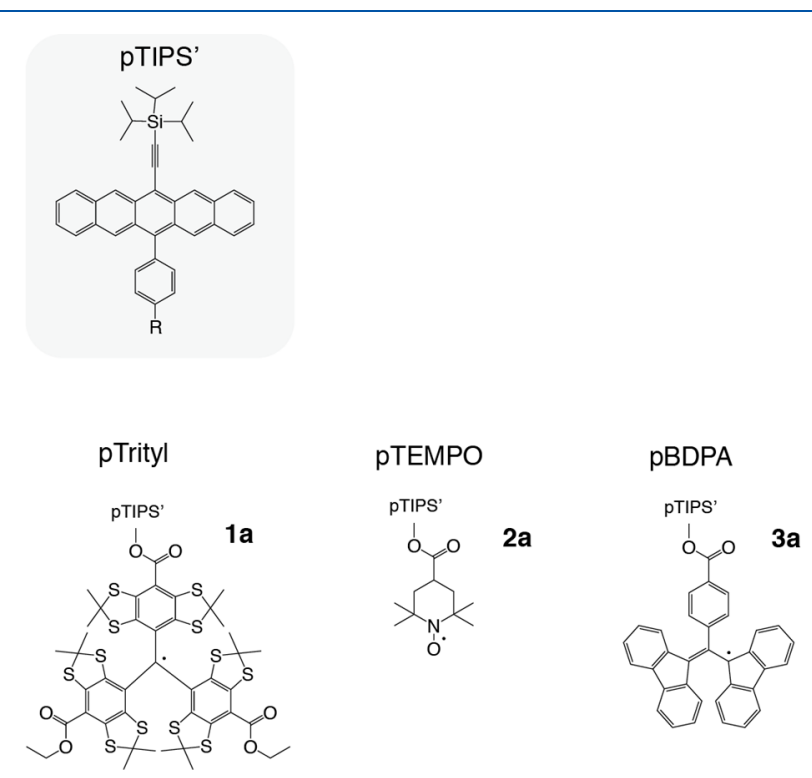

Figure 1. Pentacene derivative compounds investigated in this study. Bis(triisopropylsilylethynyl (TIPS)) pentacene bound via a linker to three different radicals.

that are functionalized with identical linkers but different stable free radicals: Triarylmethyl (Finland trityl), 1,3-bisdiphenylene2-phenylallyl (BDPA), and (2,2,6,6-tetramethylpiperidin-1yl)oxy (TEMPO).

We measured the transient EPR (TREPR) spectra of these three compounds, and we observe that only the pentacenetrityl ( $\mathrm{p}$ Trityl) derivative displays any signs of spin polarization transfer between the pentacene triplet and the tethered radical. These findings are supported by complementary transient absorption (TA) experiments which clearly show enhanced intersystem crossing (ISC). This result indicates electronic communication between the pentacene triplet and the trityl radical which is absent in the BDPA and TEMPO moieties. We attribute this effect to a larger spatial extent of the electron spin density of the trityl radical ${ }^{32-34}$ compared to the BDPA and TEMPO derivatives and the poor $\pi$-conjugation on the linker. We also observe differences in the polarization transfer behavior when the pTrityl system is dissolved in different glassy matrices. This behavior may be due to different conformations, the glass quality, and possibly aggregate formation of the pentacene derivatives in frozen solution.

\section{METHODS}

Synthesis. The synthesis and characterization of compounds 1a, 2a, and 3a are described in the Supporting Information. The numbering scheme is in line with the synthetic description given in the SI. All reactions were carried out under an inert atmosphere of argon. The compounds were purified using column chromatography on silica gel and characterized using EPR and high-resolution electrospray ionization mass spectrometry (ESI-HRMS). Further details regarding the synthesis and analysis can be found in the SI.
Sample Preparation for EPR. Butyronitrile (btn), toluene (tol), $o$-terphenyl (OTP), and dimethyl sulfoxide (DMSO) were purchased from Sigma-Aldrich and used without further purification. The samples were prepared at a concentration of approximately $0.1 \mathrm{mM}$, degassed by freezing and thawing the solution (several cycles), and subsequently sealed under vacuum in a $3.8 \mathrm{~mm}$ outer diameter EPR tube. Samples prepared with OTP were mixed with powdered OTP and then mildly heated to form a homogeneous mixture. Samples in OTP were not sealed or degassed.

Optical Spectroscopy. Compounds 1a, 2a, and 3a were dissolved in butyronitrile, and their ground-state electronic properties were probed at room temperature by steady-state UV-vis absorption spectroscopy on an Agilent Cary $60 \mathrm{UV}-$ vis spectrophotometer. The solutions were prepared to give an optical density at the excitation wavelength in the range of $0.2-$ 0.4 corresponding to a concentration of $\sim 0.1 \mathrm{mM}$. In order to investigate the excited-state dynamics of the compounds, a global kinetic analysis of the femtosecond ( $f s$ ) and nanosecond (ns) transient absorption spectra was performed. Further details regarding the experimental setups and data analysis can be found in the Supporting Information.

Electron Paramagnetic Resonance. EPR and Laser Setup. All EPR experiments were carried out on a Bruker ELEXSYS E680 spectrometer operated at the X-band frequency (9.75 GHz) using a Bruker ER4118X-MD5 resonator. The temperature was held constant at $85 \mathrm{~K}$ using a nitrogen gas-flow cryostat (Oxford Instruments). The samples were photoexcited with depolarized laser light at $620 \mathrm{~nm}$ using an OPO (Opotek Opolette 355) seeded by the third harmonic of a Nd:YAG operated at $20 \mathrm{~Hz}$ (pulse duration $5 \mathrm{~ns}$ ). Excitation energies between 1 and $4 \mathrm{~mJ}$ were used (as indicated) with a $2 \mathrm{~mm}$ beam diameter. The spectra were field corrected using a BDPA field standard $(g=2.00242)$ and frequency corrected to $9.75 \mathrm{GHz}$.

Transient CW EPR. The transient CW EPR spectra were acquired in direct detection mode using the transient recorder and a microwave power of $0.2 \mathrm{~mW}(30 \mathrm{~dB})$. Any positive signal corresponds to an absorptive transition and any negative signal to an emissive one. After data acquisition, the $2 \mathrm{D}$ spectra were baseline corrected in both dimensions using a home-written MATLAB routine.

Pulsed EPR. Transient field-swept echo-detected EPR spectra were recorded at $85 \mathrm{~K}$ using the microwave pulse sequence laser $-t_{1}-\pi / 2-\tau-\pi-\tau$-echo with $t_{1} \approx 2 \mu$ s and an interpulse delay $\tau$ of 120 ns. The delay between laser excitation and the start of the microwave pulse sequence was optimized from the maximum EPR signal enhancement using a varied delay after the flash experiment, which showed the EPR signal maximum at 2 $\mu$ s. A two-step phase cycle was applied, and integration was performed over the whole echo. However, in the case of pTrityl, the EPR line shape is sufficiently narrow such that FID detection rather than echo detection needed to be used to obtain a spectrum free of artifacts. The shot repetition rate was determined by the repetition rate of the laser $(20 \mathrm{~Hz})$. An electronic delay generator (Stanford Research Systems DG645) was used as the master clock to synchronize the laser excitation pulse with the microwave pulse sequence.

For the dark experiments, the laser beam was blocked before reaching the sample. All other experimental parameters were kept the same. All fits to the experimental data were done in MATLAB using EasySpin functions. ${ }^{38}$ 


\section{RESULTS AND DISCUSSION}

We synthesized, isolated, and characterized the molecules shown in Figure 1 according to the methods given in the Supporting Information. For the pentacene part of the molecule we used a TIPS-pentacene ${ }^{39}$ moiety as shown in Figure 1 , since the TIPS group has been shown to increase the solubility of pentacene compounds. ${ }^{39,40}$ The linker is identical for all of the pentacene-radical derivatives studied and consists of a phenyl spacer and a carboxyl moiety. The conjugation was deliberately shortened at the phenyl spacer rather than extending to the freeradical substituent in order to investigate how changing the stable radical affects the spin polarization transfer.

UV-vis Absorption Measurements. Figure 2 shows optical absorption profiles of pTEMPO, pBDPA, and pTrityl

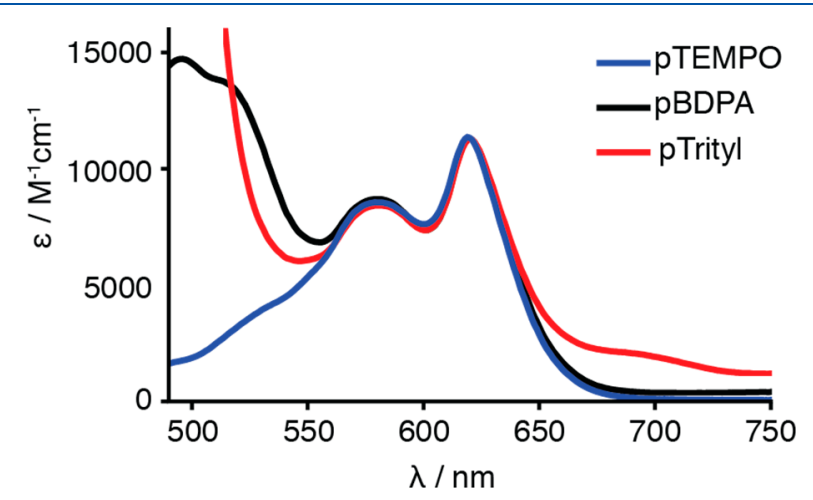

Figure 2. Optical absorption profiles of pTEMPO, pBDPA, and pTrityl radicals in butyronitrile normalized to the maximum absorption of the pentacene moiety $620 \mathrm{~nm}$, and scaled with known absorption coefficient. ${ }^{6}$ The absorption spectra of the untethered TEMPO, BDPA and trityl radicals have been previously reported. ${ }^{35-37}$

radicals in butyronitrile normalized to the maximum absorption of the pentacene moiety. The pentacene moiety in all three pentacene-radical derivatives has an absorption maximum at $\lambda$ $=620 \mathrm{~nm}$ corresponding to the $S_{0} \rightarrow S_{1}$ pentacene transition. The absorption spectra of all three pentacene-radical derivatives are very similar, showing that the different radical moieties do not have significantly different effects on the pentacene singlet electronic state energies. Similar pentacene derivatives show $\lambda_{\max }=620 \mathrm{~nm}$ when the pentacene is directly bound to a phenyl group. ${ }^{6}$ Changes in the substituents bound to the phenyl did not significantly affect the absorption profile of the compound. The trityl and BDPA moieties contribute to the strong absorption observed below $550 \mathrm{~nm}^{35}$ in Figure 2 .

Transient Absorption Spectroscopy. Excited-state electronic properties and lifetimes of pBDPA, pTrityl, and pTEMPO were probed using nanosecond (ns-TA) and femtosecond (fs-TA) transient absorption spectroscopy. The excitation wavelength was set to 620 and $600 \mathrm{~nm}$ in order to populate the lowest singlet excited state $\left(S_{1}\right)$ upon photoexcitation. The transient absorption spectra were recorded with time delays ranging from 0.1 to $1880 \mathrm{ps}$ and from 1 to $500 \mathrm{~ns}$. As shown in Figures 3 and 4, the spectra of the pBDPA and pTEMPO compounds show that even after 100 ps the spectra are dominated by a strong absorption between 430 and $445 \mathrm{~nm}$, which we assign to the excited singlet state absorption in agreement with previous work. ${ }^{6}$ This absorption decreases on a nanosecond time scale, indicative of the decay of the $S_{1}$ population. ${ }^{6}$
The spectra of the pTrityl in butyronitrile and toluene, however, show a singlet decay that is 2 orders of magnitude faster when compared to $\mathrm{pTEMPO}$ and $\mathrm{pBDPA}$. In addition, in the range of $10-100 \mathrm{ps}$, an absorption feature appears at 505 $\mathrm{nm}$. We assign this feature to the triplet state absorption $\left(\mathrm{T}_{1} \rightarrow\right.$ $\mathrm{T}_{3}$ ) which has already been reported elsewhere. ${ }^{19,42}$ The lifetimes of the singlet and triplet states of pBDPA, pTEMPO, and pTrityl are summarized in Table 1 . In the range between 1 and $500 \mathrm{~ns}$, no excited singlet state absorption is observed in the pTrityl systems (at $450 \mathrm{~nm}$ ), implying a fast decay of the $S_{1}$ population as evident from the fs-TA data. Moreover, the peak at $505 \mathrm{~nm}$, which corresponds to the lowest triplet state, shows a decay of hundreds of nanoseconds, which is considerably faster than the reported decay of other pentacene derivatives. ${ }^{19}$

Because the $S_{1}$ lifetime reported in Table 1 includes contributions due to radiative decay, intersystem crossing, as well as internal conversion, a numerical value for the intersystem crossing rate cannot be directly extracted from the $S_{1}$ lifetime. However, we find that in addition to a faster singlet decay, the onset of triplet formation in the pTrityl is much faster than that for pBDPA and pTEMPO (Table 1 and Figure S13). This correlation would be expected if the trityl radical is acting to enhance the intersystem crossing rate. Also, when compared to the pentacene derivatives reported by Ito et al., ${ }^{19} \mathrm{pBDPA}$ and pTEMPO exhibit shorter singlet lifetimes, which suggests that the BDPA and TEMPO radicals affect the dynamics of the pentacene triplet formation but not as strongly as the trityl radical. However, unlike Ito et al., ${ }^{19}$ we do not observe any evidence of singlet fission occurring in dilute pentacene derivative solutions as the $S_{1}$ decay can be fit to a first-order process. $^{42}$

A faster singlet decay could also be indicative of excitation energy transfer from the pentacene to the radical. However, we can exclude this possibility for the case of pTrityl as no signatures of the trityl excited-state absorption could be observed in the TA data. Ultimately, we observe a decrease in the singlet lifetime by 2 orders of magnitude for pTrityl when compared to the pBDPA and pTEMPO systems. As we have excluded the possibility of excitation energy transfer, this suggests that enhanced, spin-allowed ISC ${ }^{19}$ occurs for the pTrityl compound but not for pBDPA or pTEMPO.

EPR Measurements. Pulsed EPR spectra of pTrityl (1a), pTEMPO (2a), and pBDPA (3a) with and without photoillumination prior to the microwave pulse sequence at $85 \mathrm{~K}$ as well as the compound pTrityl (1a) in three different solvents are shown in Figure 5. The measurements of pTEMPO and pBDPA indicate little to no polarization transfer between the pentacene triplet and the tethered radical upon photoillumination. However, we observe clear optically induced polarization transfer in the case of the pentacene tethered to the trityl radical. We also find that the lifetime of the spin polarization on the trityl, $\sim 900 \mu \mathrm{s}$ (see Figure S16c for fit and bounds), correlates well with the spin-lattice relaxation time of pTrityl reported in Table 2. We observe an enhancement in the trityl EPR signal of $\sim 1.6$ in a mixture of 60:40 DMSO: $\mathrm{D}_{2} \mathrm{O}$, though the local enhancement is likely to be somewhat larger as the illuminated region did not cover the total detection volume of the resonator. We estimate a local enhancement of a factor $\sim 10$ using parameters discussed in the Supporting Information.

In Figure 5 it can also be seen that the degree of electron spin polarization in pTrityl depends on the glassy solvent matrix, with the largest effect being seen in DMSO: $\mathrm{D}_{2} \mathrm{O}$ and the effect essentially disappearing in toluene. It is possible that this 

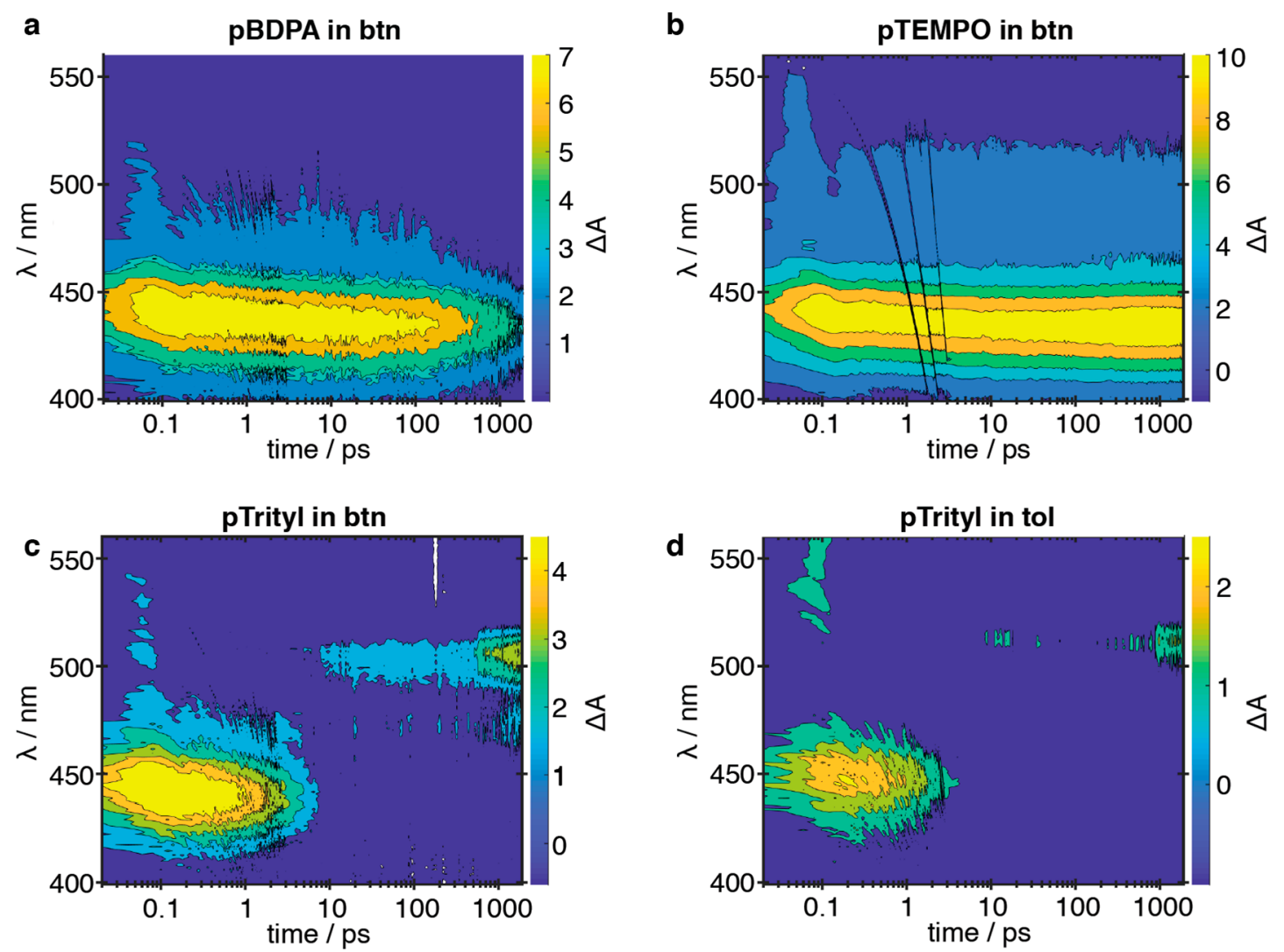

Figure 3. Transient absorption spectra on the picosecond time scale of (a) pBDPA, (b) pTEMPO, (c) pTrityl in butyronitrile, and (d) pTrityl in toluene at different time delays following $600 \mathrm{~nm}$ pulsed excitation.
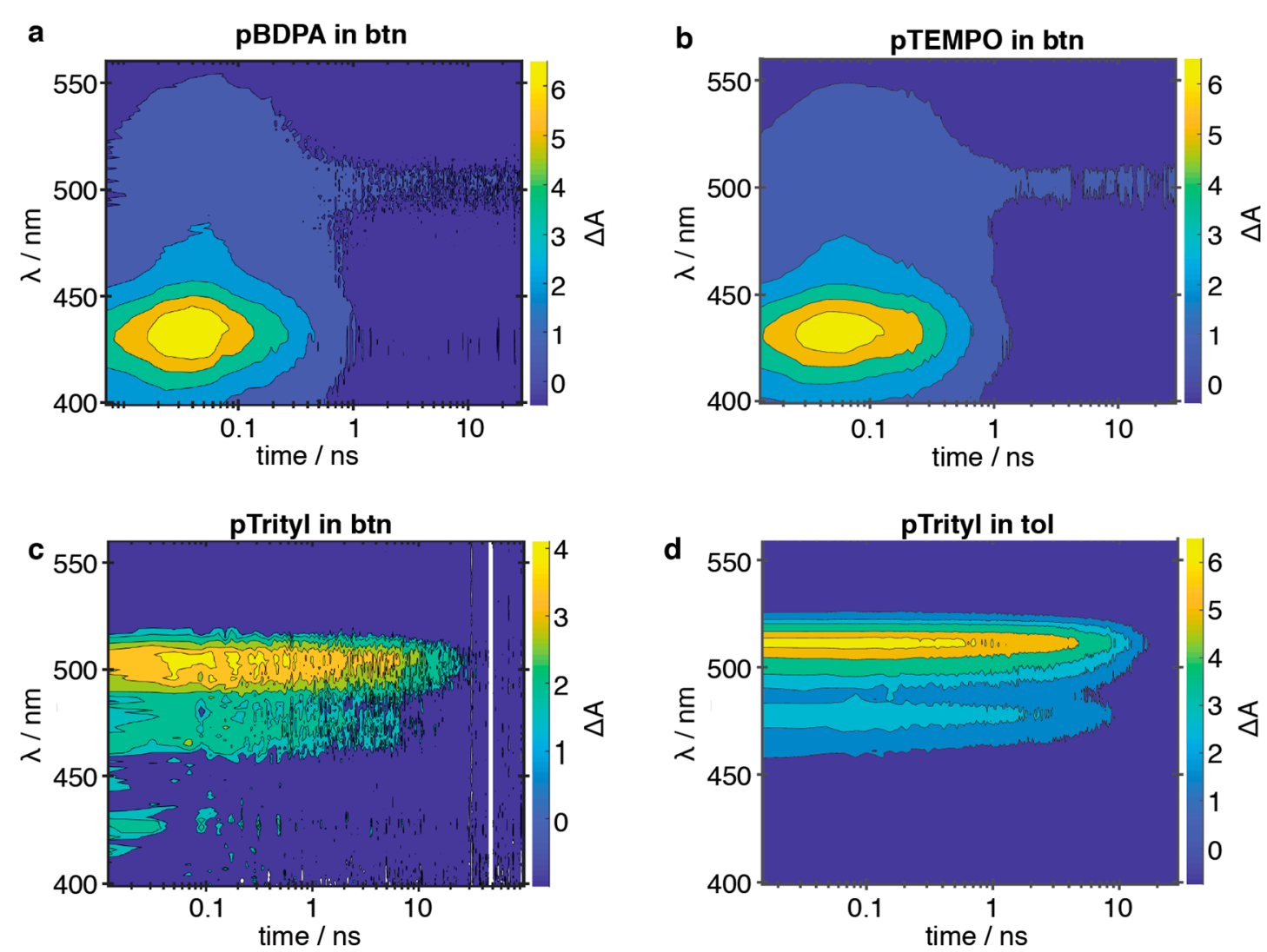

Figure 4. Transient absorption spectra on the nanosecond time scale of (a) pBDPA, (b) pTEMPO, (c) pTrityl in butyronitrile, and (d) pTrityl in toluene at different time delays following $620 \mathrm{~nm}$ pulsed excitation. 
Table 1. Summary of Singlet and Triplet Lifetimes Extracted from the TA Spectra of Figures 3 and $4^{a}$

\begin{tabular}{|c|c|c|}
\hline sample/solvent & $\mathrm{S}_{1}$ lifetime (ps) & $\mathrm{T}_{1}$ lifetime (ns) \\
\hline TIPS-pentacene $e^{41}$ & 11000 & 6500 \\
\hline pTEMPO/btn & $4300 \pm 100$ & microsecond range 6 \\
\hline $\mathrm{pBDPA} / \mathrm{btn}$ & $2800 \pm 100$ & microsecond range \\
\hline pTrityl/btn & $4.55 \pm 0.10$ & $247 \pm 20$ \\
\hline pTrityl/tol & $2.67 \pm 0.13$ & $286 \pm 4$ \\
\hline
\end{tabular}

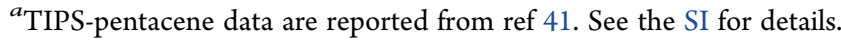

difference arises due to varying properties of the glass, which in turn would have a direct effect on the optical transparency of the sample. The distribution of frozen conformations could also play a role, as the solvents used have distinct freezing points. The distribution of conformations will depend on the accessible conformations at the freezing point in the solvent, and this can lead to cases where the chromophore and radical experience slightly distinct magnetic couplings in the different solvents. The formation of aggregates could also be a factor in this solvent effect. Transient EPR investigations of the pentacene-radical derivative precursor molecule pOTBS (see Supporting Information) as well as model compound 6,13-bis((triethylsilyl)ethynyl)pentacene (pTES) show signs of stacking/aggregation of these compounds in frozen solution. Such aggregates are known to form in solid-state films of bis(triisopropylsilylethynyl (TIPS)) pentacene as evidenced by optical measurements. ${ }^{41}$ We observe quintet signatures rather than a triplet EPR spectrum, which suggests the close proximity (i.e., coupling) of two triplet states. However, this possible aggregate formation is expected to be reduced when bulky radical substituents are attached to the chromophore.

Note that the EPR spectra in Figure 5 are all very similar to the spectra for the respective untethered free radicals. ${ }^{43-45}$ Isotropic $g$ factors in a dichloromethane solution at room temperature are shown in Table 2 as well as their respective spin-lattice relaxation lifetimes at $85 \mathrm{~K}$ in the solid-state. The $\mathrm{g}$ tensor
Table 2. EPR Fit Parameters: Spin-Lattice electron Relaxation Lifetimes $\left(T_{1}\right)$ at the X-Band for pTEMPO, pBDPA, and pTrityl-Tethered Free Radicals at $85 \mathrm{~K}$ in Butyronitrile and Isotropic $g$ Factors at Room Temperature in Dichloromethane ${ }^{a}$

$\begin{array}{lcc}\text { compound } & g_{\text {iso }} & T_{1}(\mathrm{~ms}) \\ \text { pTEMPO } & 2.0062 & 0.23 \pm 0.03 \\ \text { pBDPA } & 2.0026 & 40.3 \pm 0.6 \\ \text { pTrityl } & 2.0030 & 1.07 \pm 0.01\end{array}$

${ }^{a}$ The corresponding fitted $\mathbf{g}$ tensor values in the solid state are reported in the SI.

components for the three compounds at $85 \mathrm{~K}$ in butyronitrile are reported in Table S1.

In order to further explore the mechanism of polarization transfer between the pentacene triplet and the trityl radical, transient EPR (TREPR) experiments were run on frozen solutions at $85 \mathrm{~K}$. The TREPR spectra of pTrityl at different time delays after photoexcitation are shown in Figure 6. As can be

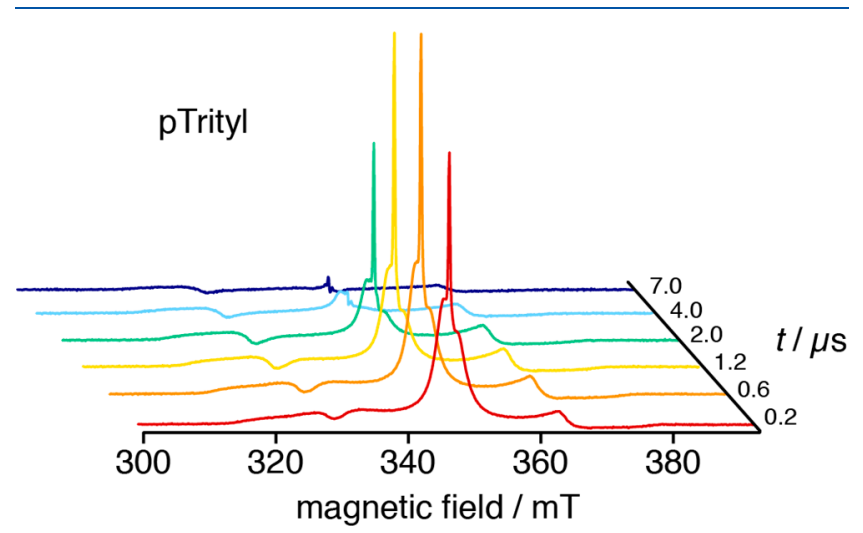

Figure 6. Transient cw EPR spectra of $0.1 \mathrm{mM}$ pTrityl in butyronitrile at $85 \mathrm{~K}$ taken at different time delays after laser excitation.
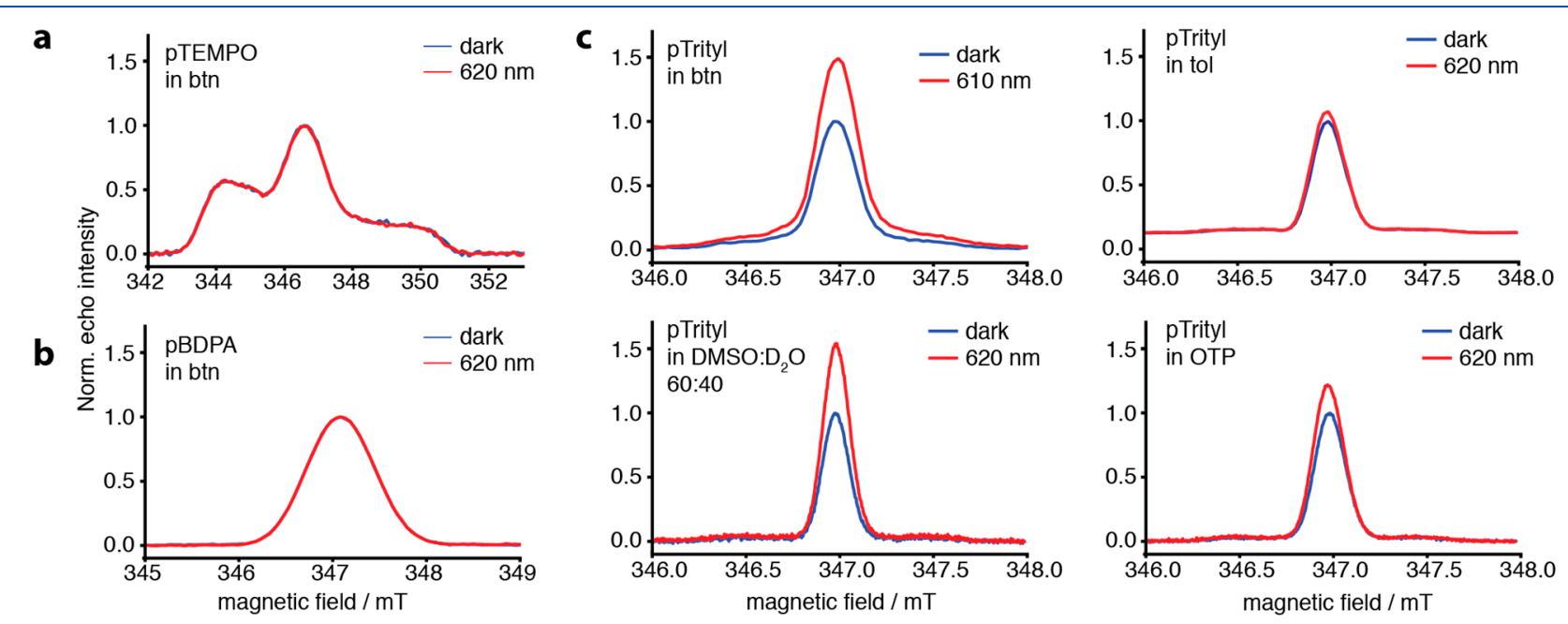

Figure 5. Pulse EPR spectra taken at $85 \mathrm{~K}$ at $9.75 \mathrm{GHz}$ (X-band) with and without a $5 \mathrm{~ns}$ photoexcitation pulse before the measurement: (a) $0.1 \mathrm{mM}$ pTEMPO in butyronitrile (btn), (b) $0.1 \mathrm{mM} \mathrm{pBDPA}$ in btn, (c) $0.1 \mathrm{mM}$ pTrityl in four different solvents: btn, $60 \%$ dimethyl sulfoxide (DMSO) and $40 \% \mathrm{D}_{2} \mathrm{O}$, o-terphenyl (OTP), and toluene (tol). "Dark" spectra were given a relative intensity of 1.0; therefore, the $y$ axis yields the electron polarization enhancement factor when comparing the dark and light spectra. All spectra obtained following photoexcitation in btn were taken with $4 \mathrm{~mJ}$ beam energy with a $5 \mathrm{~ns}, 620$ or $610 \mathrm{~nm}$ laser pulse followed by a $2 \mu$ s delay before acquisition. All other spectra with light were taken with a $1 \mathrm{~mJ}$ beam energy at $620 \mathrm{~nm}$ with the same delay after the laser pulse. Size of the beam on the sample was $\sim 2 \mathrm{~mm}$ in diameter, and volume detected by EPR was approximately $70 \mu \mathrm{L}$. For reference, the optical density of our samples at these concentrations is $\sim 0.3$ based on the path length of the sample and the molar absorptivity at the excitation wavelength for similar compounds. ${ }^{6}$ 
seen from the figure, not all spectral features exhibit the same decay behavior, suggesting that they originate from different species. The spectra shown in Figure 6 are characterized by a sharp positive EPR signal associated with the trityl radical at $\sim 348 \mathrm{mT}$ along with some broader, absorptive, and less intense features underneath this peak in the range of 342-350 mT, which might be associated with a quartet state. The spectra also contain a broad feature with absorptive and emissive peaks in the range from 320 to $370 \mathrm{mT}$. The absorptive and emissive features in the region of $320-370 \mathrm{mT}$ could be simulated as a triplet state using EasySpin with zero-field parameters $D \approx 1270 \mathrm{MHz}$ and $E$ $\approx-100 \mathrm{MHz}$ and $\mathbf{g}$-tensor values reported for pentacene. ${ }^{46}$ These values correlate reasonably well with EPR parameters for triplet states formed in pentacene in a naphthalene matrix ${ }^{46}$ where the reported $\mathbf{g}$ tensor is [2.0015, 2.0009, 2.0005], with zero-field splitting parameters $D=1389 \mathrm{MHz}$ and $E=-42$ $\mathrm{MHz}$.

The mechanism of polarization transfer in such systems depends on spin relaxation processes, excited-state energetics, as well as the magnetic coupling regime, which depends on the magnitude of the exchange interaction between the excited triplet and the tethered radical compared to other magnetic interactions in the system. We observe a spin-polarized groundstate radical signature in the TREPR spectra (Figure 6), which indicates that the spin-spin interaction in some of the pTrityl molecules falls within the weak coupling regime. However, additional features in the TREPR spectrum underneath the sharp trityl feature could be indicative of an intermediate/strong coupling case. We propose that these features could arise from quartet state formation. Quartet features are expected to be narrower than the pure triplet for the case where the dipolar coupling between unpaired electrons in the triplet is stronger than that between the pentacene triplet and the free-radical doublet. ${ }^{47}$ In an intermediate coupling regime, the degree of mixing between the pentacene triplet and the free-radical doublet would be dependent on the molecular orientation with respect to the local magnetic field. ${ }^{47}$ Because of the rotation and reduced conjugation of the linker in pTrityl the individual molecules may be frozen in different conformations which lead to a distribution of magnetic couplings between the pentacene triplet and the tethered radical. An intermediate coupling regime could also explain the observation of a triplet signature in the TREPR spectrum as this could arise due to very weak coupling between the free-radical doublet and the pentacene triplet states for a particular orientation of pTrityl. The formation of triplet states could also arise from a small concentration of degraded pTrityl, although no significant change in the pTrityl EPR signal was observed over the course of multiple measurements. We note that additional TREPR measurements performed at higher microwave frequencies could potentially help to clarify the assignment of the spectral features, particularly with respect to quartet state formation. However, no such measurements were carried out for this study.

The electron spin polarization produced on a free-radical that is tethered to a chromophore could arise from different mechanisms depending on the coupling regime. ${ }^{6,29}$ As pTrityl is likely to be in an intermediate regime, it is possible that a couple of these mechanisms are involved in the transfer. For the case of the electron spin polarization transfer (ESPT) mechanism, non-Boltzmann spin polarization is transferred from the excited triplet to the neighboring radical via weak spin exchange interactions. This would lead to a strong spinpolarized signal on the radical, which is indeed what is observed in Figures 5 and 6. Within the framework of the reverse quartet pair mechanism (RQPM), strong exchange interactions between the radical and the triplet lead to mixing between doublet and quartet excited states. This results in selective relaxation between an excited doublet state and the doublet ground state, thereby polarizing the tethered free radical. ${ }^{25}$ There is some indication of quartet formation in the TREPR measurements in Figure 6; however, we observe no polarization inversion of the electron spin polarization on the trityl radical over time, which would be a typical signature of the RQPM.

Of the three radicals used in this study, only the pentacenetrityl system resulted in observable electron spin polarization after photoexcitation of the pentacene chromophore. In order to make sense of these results, it is informative to discuss the spin density distributions on the free radicals trityl, TEMPO, and BDPA. Density functional theory (DFT) calculations estimating the spin delocalization on an untethered trityl radical ${ }^{33}$ have shown that the majority of the spin density lies on the central carbon atom (70\%) with the remainder distributed across the phenyl group carbons (26\%) and carboxyl groups (4\%). Comparing similar DFT and EPR studies done on the TEMPO and BDPA free radicals, ${ }^{34}$ for TEMPO nearly all ( $\sim 90 \%)$ of the electron spin density lies on the nitrogen and oxygen atoms while on the $\mathrm{BDPA}^{32}$ it is primarily on the carbon near the fluorenyl ring with very low spin density spread over the $\beta$-aryl group. Thus, compared to BDPA and TEMPO, the electron spin density of the trityl radical is spread over a larger spatial extent, which could explain why coupling between the pentacene triplet and the trityl radical is still observed despite poor conjugation of the chromophore-radical linker.

\section{CONCLUSIONS}

The findings here are an important step toward understanding the mechanisms underlying the excited-state dynamics in pentacene-radical systems. We investigated the electronic and spin properties of three pentacene derivatives tethered to TEMPO, BDPA, and trityl radicals and found that the rate of intersystem crossing is significantly enhanced for the pTrityl system but not for pTEMPO and pBDPA. The excited triplet state lifetime of pTrityl is an order of magnitude shorter than that of pentacene alone, indicative of efficient triplet quenching. By transient EPR spectroscopy we could further show that the electron spin polarization of the excited triplet state of pentacene is transferred to the tethered stable radical in pTrityl, resulting in an intensity change of the transient EPR signal by a factor of 2 . The observation of electron spin polarization transfer solely for pTrityl may be related to an increased spatial extent of the trityl spin density as compared to the TEMPO and BDPA free radicals, leading to a larger value for the exchange coupling between the pentacene and the stable radical moieties.

\section{ASSOCIATED CONTENT}

\section{SI Supporting Information}

The raw data reported here are available at http://dx.doi.org/ 10.5281 /zenodo.3755735. The Supporting Information is available free of charge at https://pubs.acs.org/doi/10.1021/ acs.jpca.0c03498.

Additional information on synthesis and characterization (NMR, EPR, ESI-HRMS, TA) of the compounds used in this study (PDF) 


\section{AUTHOR INFORMATION}

\section{Corresponding Authors}

Olivier Ouari - Aix Marseille Univ, CNRS, ICR, Marseille, France; (1) orcid.org/0000-0003-4320-4313; Email: olivier.ouari@univ-amu.fr

Lyndon Emsley - Institut des Sciences et Ingénierie Chimiques, Ecole Polytechnique Féderale de Lausanne (EPFL), CH-1015 Lausanne, Switzerland; (i) orcid.org/0000-0003-1360-2572; Email: lyndon.emsley@epfl.ch

\section{Authors}

Claudia E. Avalos - Institut des Sciences et Ingénierie Chimiques, Ecole Polytechnique Féderale de Lausanne (EPFL), CH-1015 Lausanne, Switzerland

Sabine Richert - Centre for Advanced Electron Spin Resonance (CAESR), University of Oxford, OX1 3QR Oxford, United Kingdom; (i) orcid.org/0000-0002-2990-974X

Etienne Socie - Institut des Sciences et Ingénierie Chimiques, Ecole Polytechnique Féderale de Lausanne (EPFL), CH-1015 Lausanne, Switzerland

Ganesan Karthikeyan - Aix Marseille Univ, CNRS, ICR, Marseille, France

Gilles Casano - Aix Marseille Univ, CNRS, ICR, Marseille, France

Gabriele Stevanato - Institut des Sciences et Ingénierie Chimiques, Ecole Polytechnique Féderale de Lausanne (EPFL), CH-1015 Lausanne, Switzerland; (i) orcid.org/0000-00030020-1286

Dominik J. Kubicki - Institut des Sciences et Ingénierie Chimiques, Ecole Polytechnique Féderale de Lausanne (EPFL), CH-1015 Lausanne, Switzerland; 이이.org/0000-00029231-6779

Jacques E. Moser - Institut des Sciences et Ingénierie Chimiques, Ecole Polytechnique Féderale de Lausanne (EPFL), CH-1015 Lausanne, Switzerland; (1) orcid.org/0000-0003-0747-4666

Christiane R. Timmel - Centre for Advanced Electron Spin Resonance (CAESR), University of Oxford, OX1 3QR Oxford, United Kingdom; (1) orcid.org/0000-0003-1828-7700

Moreno Lelli - Center of Magnetic Resonance (CERM) and Department of Chemistry "Ugo Schiff", University of Florence, 50019 Sesto Fiorentino, Italy; (1) orcid.org/0000-0002-70422335

Aaron J. Rossini - Institut des Sciences et Ingénierie Chimiques, Ecole Polytechnique Féderale de Lausanne (EPFL), CH-1015 Lausanne, Switzerland; 이이.org/0000-0002-1679-9203

Complete contact information is available at:

https://pubs.acs.org/10.1021/acs.jpca.0c03498

\section{Author Contributions}

The manuscript was written through contributions of all authors. All authors have given approval to the final version of the manuscript.

\section{Notes}

The authors declare no competing financial interest.

\section{ACKNOWLEDGMENTS}

The authors thank Yifan Quan and Patrick Hautle at the Paul Scherer Institute for assistance with preliminary experiments. This work was supported by Swiss National Science Foundation Grant No. 200020178860 and by the CAESR facility in Oxford under the EPSRC grant (EPL011972/1). G.S. acknowledges Marie-Skłodowska-Curie Grant 796904.

\section{ABBREVIATIONS}

NMR, nuclear magnetic resonance; ESR, electron spin resonance; TEMPO, (2,2,6,6-tetramethylpiperidin-1-yl)oxyl; BDPA, $\alpha, \gamma$-bisdiphenylene- $\beta$-phenylallyl

\section{REFERENCES}

(1) Guo, H.; Peng, Q.; Chen, X.-K.; Gu, Q.; Dong, S.; Evans, E. W.; Gillett, A. J.; Ai, X.; Zhang, M.; Credgington, D.; Coropceanu, V.; Friend, R. H.; Bredas, J.-L.; Li, F.; et al. High Stability and Luminescence Efficiency in Donor-Acceptor Neutral Radicals Not Following the Aufbau Principle. Nat. Mater. 2019, 18, 977-984.

(2) Peng, Q. M.; Obolda, A.; Zhang, M.; Li, F. Organic Light-Emitting Diodes Using a Neutral Pi Radical as Emitter: The Emission from a Doublet. Angew. Chem., Int. Ed. 2015, 54, 7091-7095.

(3) Ai, X.; Evans, E. W.; Dong, S. Z.; Gillett, A. J.; Guo, H. Q.; Chen, Y. X.; Hele, T. J. H.; Friend, R. H.; Li, F. Efficient Radical-Based LightEmitting Diodes with Doublet Emission. Nature 2018, 563, 536-540.

(4) Morita, Y.; Suzuki, S.; Fukui, K.; Nakazawa, S.; Kitagawa, H.; Kishida, H.; Okamoto, H.; Naito, A.; Sekine, A.; Ohashi, Y.; et al. Thermochromism in an Organic Crystal Based on the Coexistence of Sigma- and Pi-Dimers. Nat. Mater. 2008, 7, 48-51.

(5) Simao, C.; Mas-Torrent, M.; Crivillers, N.; Lloveras, V.; Artes, J. M.; Gorostiza, P.; Veciana, J.; Rovira, C. A Robust Molecular Platform for Non-Volatile Memory Devices with Optical and Magnetic Responses. Nat. Chem. 2011, 3, 359-364.

(6) Chernick, E. T.; Casillas, R.; Zirzlmeier, J.; Gardner, D. M.; Gruber, M.; Kropp, H.; Meyer, K.; Wasielewski, M. R.; Guldi, D. M.; Tykwinski, R. R. Pentacene Appended to a Tempo Stable Free Radical: The Effect of Magnetic Exchange Coupling on Photoexcited Pentacene. J. Am. Chem. Soc. 2015, 137, 857-863.

(7) Buchachenko, A. L.; Berdinsky, V. L. Electron Spin Catalysis. Chem. Rev. 2002, 102, 603-612.

(8) Kobori, Y.; Takeda, K.; Tsuji, K.; Kawai, A.; Obi, K. Exchange Interaction in Radical-Triplet Pairs: Evidences for CIDEP Generation by Level Crossings in Triplet-Doublet Interactions. J. Phys. Chem. A 1998, 102, 5160-5170.

(9) Goudsmit, G. H.; Paul, H.; Shushin, A. I. Electron-Spin Polarization in Radical Triplet Pairs - Size and Dependence on Diffusion. J. Phys. Chem. 1993, 97, 13243-13249.

(10) Krumkacheva, O. A.; Timofeev, I. O.; Politanskaya, L.; Polienko, Y. F.; Tretyakov, E. V.; Rogozhnikova, O. Y.; Trukhin, D. V.; Tormyshev, V. M.; Chubarov, A. S.; Bagryanskaya, E. G.; et al. Triplet Fullerenes as Prospective Spin Labels for Nanoscale Distance Measurements by Pulsed Dipolar EPR Spectroscopy. Angew. Chem. 2019, 131, 13405-13409.

(11) Coppo, P.; Yeates, S. G. Shining Light on a Pentacene Derivative: The Role of Photoinduced Cycloadditions. Adv. Mater. 2005, 17, 3001-3005.

(12) Colvin, M. T.; Carmieli, R.; Miura, T.; Richert, S.; Gardner, D. M.; Smeigh, A. L.; Dyar, S. M.; Conron, S. M.; Ratner, M. A.; Wasielewski, M. R. Electron Spin Polarization Transfer from Photogenerated Spin-Correlated Radical Pairs to a Stable Radical Observer Spin. J. Phys. Chem. A 2013, 117, 5314-5325.

(13) Rozenshtein, V.; Berg, A.; Stavitski, E.; Levanon, H.; Franco, L.; Corvaja, C. Electron Spin Polarization of Functionalized Fullerenes. Reversed Quartet Mechanism. J. Phys. Chem. A 2005, 109, 1114411154.

(14) Sartori, E.; Toffoletti, A.; Corvaja, C.; Garlaschelli, L. Electron Spin Polarization Transfer and Radical-Triplet Pair Polarization in Nitroxide-C-60 Derivative Systems. J. Phys. Chem. A 2001, 105, 10776-10780.

(15) Rane, V.; Das, R. Distance Dependence of Electron Spin Polarization During Photophysical Quenching of Excited Naphthalene by Tempo Radical. J. Phys. Chem. A 2015, 119, 5515-5523.

(16) Wang, Z.; Gao, Y.; Hussain, M.; Kundu, S.; Rane, V.; Hayvali, M.; Yildiz, E. A.; Zhao, J.; Yaglioglu, H. G.; Das, R.; Luo, L.; Li, J.; et al. Efficient Radical-Enhanced Intersystem Crossing in an Ndi-Tempo 
Dyad: Photophysics, Electron Spin Polarization, and Application in Photodynamic Therapy. Chem. - Eur. J. 2018, 24, 18663-18675.

(17) Colvin, M. T.; Smeigh, A. L.; Giacobbe, E. M.; Conron, S. M. M.; Ricks, A. B.; Wasielewski, M. R. Ultrafast Intersystem Crossing and Spin Dynamics of Zinc Meso-Tetraphenylporphyrin Covalently Bound to Stable Radicals. J. Phys. Chem. A 2011, 115, 7538-7549.

(18) Horwitz, N. E.; Phelan, B. T.; Nelson, J. N.; Mauck, C. M.; Krzyaniak, M. D.; Wasielewski, M. R. Spin Polarization Transfer from a Photogenerated Radical Ion Pair to a Stable Radical Controlled by Charge Recombination. J. Phys. Chem. A 2017, 121, 4455-4463.

(19) Ito, A.; Shimizu, A.; Kishida, N.; Kawanaka, Y.; Kosumi, D.; Hashimoto, H.; Teki, Y. Excited-State Dynamics of Pentacene Derivatives with Stable Radical Substituents. Angew. Chem., Int. Ed. 2014, 53, 6715-6719.

(20) Maliakal, A.; Raghavachari, K.; Katz, H.; Chandross, E.; Siegrist, T. Photochemical Stability of Pentacene and a Substituted Pentacene in Solution and in Thin Films. Chem. Mater. 2004, 16, 4980-4986.

(21) Kawanaka, Y.; Shimizu, A.; Shinada, T.; Tanaka, R.; Teki, Y. Using Stable Radicals to Protect Pentacene Derivatives from Photodegradation. Angew. Chem., Int. Ed. 2013, 52, 6643-6647.

(22) Shimizu, A.; Ito, A.; Teki, Y. Photostability Enhancement of the Pentacene Derivative Having Two Nitronyl Nitroxide Radical Substituents. Chem. Commun. 2016, 52, 2889-2892.

(23) Hetzer, C.; Basel, B. S.; Kopp, S. M.; Hampel, F.; White, F. J.; Clark, T.; Guldi, D. M.; Tykwinski, R. R. Chromophore Multiplication to Enable Exciton Delocalization and Triplet Diffusion Following Singlet Fission in Tetrameric Pentacene. Angew. Chem., Int. Ed. 2019, $58,15263-15267$.

(24) Takeda, K. Studies on Dynamic Nuclear Polarization Using Photo-Excited Triplet Electron Spins. Ph.D. Thesis, Kyoto University, March 2003.

(25) Kouno, H.; Orihashi, K.; Nishimura, K.; Kawashima, Y.; Tateishi, K.; Uesaka, T.; Kimizuka, N.; Yanai, N. Triplet Dynamic Nuclear Polarization of Crystalline Ice Using Water-Soluble Polarizing Agents. Chem. Commun. 2020, 56, 3717-3720.

(26) Tateishi, K.; Negoro, M.; Kagawa, A.; Uesaka, T.; Kitagawa, M. Hyperpolarization of Thin Films with Dynamic Nuclear Polarization Using Photoexcited Triplet Electrons. J. Phys. Soc. Jpn. 2013, 82, No. 084005 .

(27) Tateishi, K.; Negoro, M.; Kagawa, A.; Kitagawa, M. Dynamic Nuclear Polarization with Photoexcited Triplet Electrons in a Glassy Matrix. Angew. Chem., Int. Ed. 2013, 52, 13307-13310.

(28) Kundu, S.; Rane, V. Design and Photo-Induced Dynamics of Radical-Chromophore Adducts with One- or Two-Atom Separation: Toward Potential Probes for High Field Optical Dnp Experiments. J. Phys. Chem. B 2020, 124, 3163-3179.

(29) Tripathi, A.; Rane, V. Toward Achieving the Theoretical Limit of Electron Spin Polarization in Covalently Linked Radical-Chromophore Dyads. J. Phys. Chem. B 2019, 123, 6830-6841.

(30) Jenks, W. S.; Turro, N. J. Electron-Spin Polarization Transfer between Radicals. J. Am. Chem. Soc. 1990, 112, 9009-9011.

(31) Tripathi, A. K.; Rane, V.; Kundu, S.; Das, R. A Phenomenological Scheme for Reversed Quartet Mechanism of Electron Spin Polarization in Covalently Linked Systems of Chromophore and Free Radical: Determination of Magnitude of Polarization and Application to PyreneTempo Linked Molecules. J. Chem. Phys. 2019, 151, 154305.

(32) Haeri, H. H.; Spindler, P.; Plackmeyer, J.; Prisner, T. Double Quantum Coherence ESR Spectroscopy and Quantum Chemical Calculations on a BDPA Biradical. Phys. Chem. Chem. Phys. 2016, 18, 29164-29169.

(33) Kunjir, N. C.; Reginsson, G. W.; Schiemann, O.; Sigurdsson, S. Th. Measurements of Short Distances between Trityl Spin Labels with CW EPR, DQC and PELDOR. Phys. Chem. Chem. Phys. 2013, 15, 19673-19685.

(34) Lalevée, J.; Allonas, X.; Jacques, P. Electronic Distribution and Solvatochromism Investigation of a Model Radical (2,2,6,6-Tetramethylpiperidine N-Oxyl: TEMPO) through TD-DFT Calculations Including PCM Solvation. J. Mol. Struct.: THEOCHEM 2006, 767, 143-147.
(35) Rogozhnikova, O. Y.; Vasiliev, V. G.; Troitskaya, T. I.; Trukhin, D. V.; Mikhalina, T. V.; Halpern, H. J.; Tormyshev, V. M. Generation of Trityl Radicals by Nucleophilic Quenching of Tris(2,3,5,6Tetrathiaaryl)Methyl Cations and Practical and Convenient LargeScale Synthesis of Persistent Tris(4-Carboxy-2,3,5,6-Tetrathiaaryl)Methyl Radical. Eur. J. Org. Chem. 2013, 2013, 3347-3355.

(36) Lumata, L.; Ratnakar, S. J.; Jindal, A.; Merritt, M.; Comment, A.; Malloy, C.; Sherry, A. D.; Kovacs, Z. BDPA: An Efficient Polarizing Agent for Fast Dissolution Dynamic Nuclear Polarization NMR Spectroscopy. Chem. - Eur. J. 2011, 17, 10825-10827.

(37) Yang, C. Z.; Fontaine, O.; Tarascon, J. M.; Grimaud, A. Chemical Recognition of Active Oxygen Species on the Surface of Oxygen Evolution Reaction Electrocatalysts. Angew. Chem., Int. Ed. 2017, 56, 8652-8656.

(38) Walker, B. J.; Musser, A. J.; Beljonne, D.; Friend, R. H. Singlet Exciton Fission in Solution. Nat. Chem. 2013, 5, 1019-1024.

(39) Stoll, S.; Schweiger, A. Easyspin, a Comprehensive Software Package for Spectral Simulation and Analysis in EPR. J. Magn. Reson. 2006, 178, 42-55.

(40) Anthony, J. E.; Brooks, J. S.; Eaton, D. L.; Parkin, S. R. Functionalized Pentacene: Improved Electronic Properties from Control of Solid-State Order. J. Am. Chem. Soc. 2001, 123, 9482-9483.

(41) Park, S. K.; Jackson, T. N.; Anthony, J. E.; Mourey, D. A. High Mobility Solution Processed 6,13-Bis(Triisopropyl-Silylethynyl) Pentacene Organic Thin Film Transistors. Appl. Phys. Lett. 2007, 91, No. 063514.

(42) Hellner, C.; Lindqvist, L.; Roberge, P. C. Absorption-Spectrum and Decay Kinetics of Triplet Pentacene in Solution, Studied by FlashPhotolysis. J. Chem. Soc., Faraday Trans. 2 1972, 68, 1928-1937.

(43) Trukhan, S. N.; Yudanov, V. F.; Tormyshev, V. M.; Rogozhnikova, O. Y.; Trukhin, D. V.; Bowman, M. K.; Krzyaniak, M. D.; Chen, H.; Martyanov, O. N. Hyperfine Interactions of Narrow-Line Trityl Radical with Solvent Molecules. J. Magn. Reson. 2013, 233, 2936.

(44) Dupeyre, R. M.; Lemaire, H.; Rassat, A. Nitroxides. XIV. A Stable Biradical in Nitroxide Series. J. Am. Chem. Soc. 1965, 87, 3771-3772.

(45) Dane, E. L.; Swager, T. M. Synthesis of a Water-Soluble 1,3Bis(Diphenylene)2-Phenylallyl Radical. J. Org. Chem. 2010, 75, 35333536.

(46) Vanstrien, A. J.; Schmidt, J. An Electron-Paramagnetic-Res Study of the Triplet-State of Pentacene by Electron Spin-Echo Techniques and Laser Flash Excitation. Chem. Phys. Lett. 1980, 70, 513-517.

(47) Asano-Someda, M.; Toyama, N.; Kaizu, Y. Time-Resolved Epr Spectra of a Photoexcited Phenanthrene-Linked Copper(Ii)-Free-Base Porphyrin Dimer: An Intermediate-Coupling Case in a Triplet-Doublet Spin System. Appl. Magn. Reson. 2003, 23, 393-407. 